\title{
Philosophiques
}

\section{Les fonctions normatives ou catégories modales}

\section{Paul Amselek}

Volume 33, numéro 2, automne 2006

URI : https://id.erudit.org/iderudit/013889ar

DOI : https://doi.org/10.7202/013889ar

Aller au sommaire du numéro

Éditeur(s)

Société de philosophie du Québec

ISSN

0316-2923 (imprimé)

1492-1391 (numérique)

Découvrir la revue

Citer cet article

Amselek, P. (2006). Les fonctions normatives ou catégories modales. Philosophiques, 33(2), 391-418. https://doi.org/10.7202/013889ar

\section{Résumé de l'article}

Les modalités déontiques articulées par les règles de conduite en général et les règles juridiques en particulier ont constamment donné lieu à des conceptions aberrantes dans la théorie générale de l'éthique et du droit. Cette étude dénonce les vues confuses répandues en la matière par Kelsen, ainsi que les vues négationnistes tenaces en circulation prétendant purement et simplement bouter les catégories modales hors de la théorie juridique ou morale. d'utilisation que vous pouvez consulter en ligne.

https://apropos.erudit.org/fr/usagers/politique-dutilisation/ 


\title{
Les fonctions normatives ou catégories modales
}

\author{
PAUL AMSELEK \\ Professeur émérite à l'Université Panthéon-Assas (Paris II) \\ paulamselek@wanadoo.fr
}

\begin{abstract}
RÉSUMÉ. - Les modalités déontiques articulées par les règles de conduite en général et les règles juridiques en particulier ont constamment donné lieu à des conceptions aberrantes dans la théorie générale de l'éthique et du droit. Cette étude dénonce les vues confuses répandues en la matière par Kelsen, ainsi que les vues négationnistes tenaces en circulation prétendant purement et simplement bouter les catégories modales hors de la théorie juridique ou morale.
\end{abstract}

\begin{abstract}
The deontic modalities working with the rules of conduct in a general way, and the legal rules in particular, have steadily given rise to aberrant conceptions in the general theory of ethics and law. This paper informs against the confused views spread on this matter by Kelsen, and also against the circulating tenacious negationnistic views purely and simply claiming to drive modal categories out of legal or moral theory.
\end{abstract}

«Ce n'est point le corps des lois que je cherche, mais leur âme. » Montesquieu, L'esprit des lois

Une formule célèbre de Portalis définit la vocation des normes juridiques, et plus généralement de toutes les règles de conduite ou normes éthiques: « La loi ordonne, permet ou interdit. » Ordonner, permettre, interdire, c'est ce que les théoriciens du droit et de l'éthique, en particulier Kelsen, appellent les fonctions de la norme ou fonctions normatives. En logique déontique, par référence aux usages terminologiques de la logique générale, on parle plutôt des «modes ou modalités déontiques ». Mon propos ici sera de dénoncer les conceptions erronées auxquelles ces fonctions normatives ou modalités déontiques ont donné lieu et qui continuent d'avoir cours dans la théorie générale du droit et de l'éthique. Mais auparavant, il me paraît indispensable de commencer par rappeler, en manière de prolégomènes, quelques données de base relatives à cette matière.

\section{Prolégomènes}

L'idée même de "fonction normative » renvoie à la nature la plus essentielle des règles ou normes (termes qui sont pour moi rigoureusement synonymes): il s'agit d'outils, comme l'atteste la dérivation métaphorique même dont ces appellations ont originairement procédé à travers le latin regula ou le grec gnômon. Un outil, c'est un objet auquel une intention humaine a assigné une certaine finalité instrumentale. En l'occurrence, l'objet dont il s'agit est un contenu de pensée; les règles sont des outils mentaux qui appartiennent au 
monde de l'intelligible et non du sensible: ce à quoi est assignée une finalité instrumentale, c'est du sens, et notamment du sens véhiculé par des énonciations écrites ou orales ${ }^{1}$.

Plus précisément, les règles - dans leur essence la plus générale commune à toutes les espèces de règles - sont des contenus de pensée auxquels est impartie la vocation instrumentale de donner la mesure du possible, d'indiquer la marge ou degré de possibilité de l'avoir lieu de certaines choses en fonction de certaines circonstances. Telle est leur utilité (leur «outilité ») la plus essentielle, qui constitue leur dénominateur commun et à quoi s'attache, par-delà leur diversité, leur identité même de règles : elles sont destinées à servir d'indicateurs du possible. À cet égard, toutes les règles, quelles qu'elles soient, se ramènent dans leur vocation instrumentale générique à trois grandes figures selon qu'elles indiquent pour telle chose ou tel type de chose:

- une marge maximale de possibilité de survenance: "dans telles circonstances, telle chose ou tel type de chose doit survenir » (l'obligation ou nécessité, c'est la marge la plus étendue de la possibilité, la marge de $100 \%$, — le degré 1 comme disent les théoriciens du calcul des probabilités, celui qui exclut toute impossibilité et équivaut ainsi au degré 0 d'impossibilité);

— une marge nulle $(0 \%)$ de possibilité de survenance : "dans telles circonstances, telle chose ou tel type de chose ne peut pas se produire » (ce degré 0 de la possibilité correspond en même temps à la marge la plus étendue au degré 1 - de l'impossibilité);

- une marge de possibilité de survenance intermédiaire entre 0 et 1 : "dans telles circonstances, telle chose ou tel type de chose peut se produire." On est là en présence, pourrait-on dire, d'un degré d'incertitude ou indétermination occurrentielle: la chose ou le type de chose en question peut survenir ou ne pas survenir. Une réduction d'incertitude est opérée avec la catégorie particulière de règles que sont les règles de caractère probabiliste qui indiquent précisément des sous-degrés de possibilité à l'intérieur de cet espace intermédiaire entre 0 et 1 , entre $0 \%$ et $100 \%$ : «dans telles circonstances, telle chose ou tel type de chose a tel pourcentage de probabilité (par exemple, 10 chances sur 100) de se produire. »

Toutes les règles, de quelque nature qu'elles soient, servent ainsi à indiquer, pour l'avoir lieu de telle ou telle chose, des marges ou degrés sur l'échelle bipolaire du possible. S'agissant plus spécialement des règles de conduite, et notamment des règles juridiques, leur vocation instrumentale spécifique est de donner à ceux à qui elles sont adressées la marge du possible à l'intérieur de laquelle doivent se tenir leurs agissements, leurs faits et gestes - la marge de manœuvre ou latitude dont ils disposent pour leurs accom-

1. Je laisserai de côté les formes d'expression non linguistiques des normes, comme par exemple les signes gestuels de l'agent de police enjoignant à des automobilistes de circuler, de s'arrêter ou de changer de direction. 
plissements : elles sont destinées à servir de support à leur volonté, à l'encadrer dans ses déterminations, dans le choix des lignes de conduite qu'elle arrête et qu'elle met à exécution. Elles sont, en ce sens, des outils de direction de la conduite humaine: elles indiquent ce qu'on peut faire, ce qu'on ne peut pas faire ou ce qu'on ne peut pas ne pas faire, c'est-à-dire des permissions, des interdictions ou des obligations, aux fins que les destinataires se règlent sur elles, ajustent en conséquence leurs comportements.

Ces trois degrés ou marges du possible que les règles en général, et les règles éthiques et juridiques en particulier, ont vocation à fixer - chacune dans le contexte de la finalité instrumentale spécifique propre à la catégorie dont elle relève - correspondent à ce qu'on nomme en logique générale des «catégories modales » ou modalités, ces catégories modales prenant dans le contexte de la fonction directive spécifique propre aux règles de conduite la couleur ou la dimension de modalités déontiques. Dans le cadre de ces prolégomènes, il importe de dissiper certaines confusions ou contresens couramment répandus au sujet de ces catégories modales; deux mises au point me paraissent devoir être formulées.

1) Une première mise au point concerne le dénombrement de ces fonctions normatives, de ces catégories modales qui s'inscrivent dans la fonction des normes. Sont-elles bien au nombre de trois? Ne sontelles pas plutôt - selon une vue encore bien ancrée chez les logiciens - au nombre de quatre?

Il convient de rappeler, en effet, que les catégories modales ont été d'abord étudiées en logique d'une manière purement abstraite, en elles-mêmes et pour elles-mêmes, indépendamment de la théorie des règles. Depuis Aristote, la logique générale distingue ainsi quatre catégories modales, qui s'articuleraient en deux couples antithétiques : l'impossible et le possible (ou non-impossible), le nécessaire et le contingent (ou non-nécessaire). Et Leibniz, qui a été le premier dans ses Éléments de droit naturel (1669 - 1671) à faire un rapprochement entre les catégories modales et les règles (notamment juridiques) de conduite, a ramené ces dernières, dans leur vocation instrumentale, à quatre grandes figures selon qu'elles indiquent l'interdit ou son contraire, le permis (droit de faire), l'obligation ou son contraire, le facultatif (droit de ne pas faire). La logique juridique, et plus généralement normative ou déontique, vit encore sur la base de cette conception. Or elle s'inspire d'une analyse erronée développée par Aristote dans l'un de ses traités de logique (De l'interprétation) et qui a ensuite prospéré et est devenue classique chez les logiciens: le carré de catégories modales évoqué par Aristote dans ce traité

2. En liaison avec des arrière-plans métaphysiques: cf. mon étude «Ontologie du droit et logique déontique », Rev. dr. publ. 1992, p. 1016. 
est, en effet, fallacieux, car ce qui n'est pas nécessaire - le « contingent» - se confond avec la catégorie du "possible». C'est qu'en raisonnant abstraitement en termes binaires, en termes de couples de notions logiques antithétiques, le Stagirite ne s'est pas rendu compte que les catégories modales expriment les degrés du possible et que ces degrés se répartissent sur une échelle à trois échelons correspondant, comme je l'ai précédemment rappelé, respectivement au degré maximum de possibilité ou nécessité, au degré nul ou impossibilité, et au degré intermédiaire ou possibilité. Ce degré intermédiaire s'oppose à chacune des deux extrémités de l'échelle: le possible s'oppose tout à la fois au nécessaire et à l'impossible. Mais cela n'autorise pas à distinguer quatre catégories : la « possibilité » et la «contingence» distinguées par Aristote constituent une seule et même catégorie envisagée simplement tantôt par rapport à la nécessité, tantôt par rapport à l'impossibilité.

En d'autres termes, les catégories modales forment non pas un carré, mais un triangle : à ce qui n'est pas possible s'oppose non seulement ce qui est possible, ce qui peut avoir lieu, mais aussi ce qui est nécessaire, ce qui doit avoir lieu; de même, au possible s'opposent à la fois l'impossible et le nécessaire, et au nécessaire le possible et l'impossible. À chacun des trois degrés s'opposent les deux autres. C'est, du reste, ce triangle qu'Aristote lui-même a développé dans Les premiers analytiques, bien que la postérité n'ait retenu que le carré du traité De l'interprétation.

En tout cas, au niveau des règles de conduite, on doit seulement s'en tenir aux trois catégories de l'obligation, de l'interdit et du permis. Le "permis » et le "facultatif» distingués depuis Leibniz par la logique déontique sont une seule et même catégorie : une chose permise est une chose qui n'est ni interdite ni obligatoire; le droit de, c'est en même temps le droit de ne pas. Il n'y a pas de prétendu " possible unilatéral » qui serait uniquement un droit de faire ou uniquement un droit de ne pas faire.

2) Une autre mise au point s'impose en réaction à une vue couramment répandue, particulièrement en logique déontique où elle a donné lieu à de bien contestables développements théoriques. Que les normes de conduite, et en particulier les normes juridiques, aient vocation à indiquer des permissions, des interdictions ou des obligations ne veut pas dire que les énoncés qui les expriment doivent nécessairement articuler des verbes ou des expressions du type "peut», "a le droit de ", "doit ", " a l'obligation de ", « ne peut pas ", " il est interdit de »... Il s'agirait là, pour les tenants de la logique des normes, de prétendus " foncteurs normatifs » ou « déontiques », qui imprimeraient aux énoncés les articulant leur fonction même - leur 
coloration - d'énoncés de normes ${ }^{3}$. En réalité, peu importe la texture formelle - le vêtement linguistique - utilisée pour l'énonciation des normes, et notamment des normes juridiques : il ne faut pas confondre l'énoncé émis par le législateur et la fonction de norme dévolue par lui au contenu de pensée énoncé (et que traduit, dans le domaine juridique, l'intitulé même de l'acte de parole accompli : « loi », " ordonnance ", " décret », etc.). La fonction ainsi assignée à ce contenu de pensée implique qu'il est chargé de servir à donner à ceux à qui s'adresse le législateur la mesure de leur possibilité d'agir, qu'il est destiné à leur indiquer ce qu'ils peuvent, ne peuvent pas ou doivent faire, et donc qu'on doit le recevoir et l'utiliser comme tel : ainsi, c'est la fonction même de norme reconnue au contenu de pensée énoncé qui va amener ceux qui le reçoivent à s'en servir comme d'un indicateur de permissions, d'obligations ou d'interdictions. Il importe peu que le législateur ait employé formellement les termes "peut », "doit", "ne peut pas»: c'est la fonction même d'encadrement des conduites reconnue au contenu de pensée énoncé qui amène de toute façon à y rechercher et à en tirer (ou à tâcher d'en tirer) par une exégèse appropriée des permissions, des obligations, des interdictions. La fonction de norme reconnue à ce qui est énoncé branche automatiquement sur un certain registre d'interprétation orienté vers une recherche, vers une extraction de droits, d'obligations, d'interdictions; c'est cette fonction qui conduit à considérer et à utiliser ce qui est énoncé comme un gisement de "pouvoir", de "ne pas pouvoir » ou de «devoir ».

Cette mise au point éclaire, en particulier, le cas de certaines règles juridiques qui ont souvent paru atypiques, un peu mystérieuses, et qui ont fait couler beaucoup d'encre pour essayer d'en rendre compte; je veux parler des normes s'énonçant sur le modèle suivant (emprunté, en l'occurrence, au droit constitutionnel où il s'illustre par excellence): "Le Président de la République est le chef des armées ", "Le Parlement vote les lois ", "Le Gouvernement conduit la politique de la nation. » Les réalistes scandinaves de l'École d'Uppsala comme Karl Olivecrona ou Tore Strömberg ${ }^{4}$ ont prétendu qu'il s'agissait là d'une variété spéciale de normes juridiques, qualifiées de "normes de compétence ", à côté des «normes de conduite " proprement dites du type "on peut, on ne peut pas ou on doit faire ceci ou cela». Cette distinction est un non-sens : toutes les normes juridiques, et plus généralement toutes les normes éthiques, sont des règles de conduite, ayant vocation à encadrer la conduite humaine. Les contenus de pensée ainsi énoncés dans les

3. Cf. ma contribution "Philosophie du droit et théorie des actes de langage ", in Théorie des actes de langage, éthique et droit, sous ma direction, P.U.F., 1986, p. 115 s.

4. Voir S. Strömholm et H.H. Vogel, Le "réalisme scandinave» dans la philosophie du droit, L.G.D.J., 1975, p. 75 s. 
textes constitutionnels constituent des outils-règles de conduite comme les autres, se prêtant par leur nature même à une recherche herméneutique et à une extraction de marges de possibilité d'agir, de droits, d'obligations, d'interdictions. Il ne faut pas s'attacher à la forme linguistique: l'important, c'est la fonction qu'on fait jouer à ce qui est énoncé.

De la même façon, on croit généralement qu'une disposition juridique est nécessairement ou impérative, ou prohibitive, ou permissive, en fonction notamment du type de verbe ou d'expression verbale — du prétendu « foncteur» - qu'elle articule; c'est inexact. Par exemple, la disposition selon laquelle «dans telles circonstances telle autorité publique peut prendre tel type de mesures après avis de tel organisme " n'est pas une disposition purement permissive, même si elle est énoncée formellement en termes de "pouvoir » : en tant qu'elle vise à fixer la marge de manœuvre de l'autorité publique en cause, elle indique tout à la fois une possibilité (prendre ou ne pas prendre les mesures en question), des impossibilités (prendre des mesures d'une autre nature que celles prévues, ou, dans d'autres circonstances que celles mentionnées, ce qui correspond à l'obligation de ne prendre que des mesures du type indiqué et seulement dans les circonstances indiquées), et une obligation (prendre l'avis de tel organisme avant d'édicter les mesures arrêtées, ce qui correspond à l'impossibilité d'édicter ces mesures sans avoir pris l'avis de cet organisme). On le voit bien à nouveau à travers cet exemple : ce qui compte, ce n'est pas la présence formelle des verbes «devoir » ou "pouvoir » dans l'énoncé, mais le rôle même de règle que l'on assigne et que l'on fait jouer à ce qui est énoncé et qui le constitue ipso facto en gisement de droits et d'obligations aux yeux de ceux qui ont à l'interpréter et à s'en servir.

Ces mises au point faites, je suis en mesure de développer les observations critiques qu'appellent des conceptions couramment admises dans la théorie générale du droit et de l'éthique au sujet des catégories modales impliquées par les règles de conduite en général et les règles juridiques en particulier. J'examinerai d'abord, à titre emblématique, les vues confuses répandues en la matière par Kelsen, puis les vues négationnistes tenaces en circulation prétendant purement et simplement bouter les catégories modales hors de la théorie juridique ou morale.

\section{Les vues confuses répandues par Kelsen}

Il y aurait, en vérité, beaucoup à dire - et à redire — au sujet des idées développées par Kelsen autour des catégories modales dans le cadre de sa théorie du droit. Pour m'en tenir à l'essentiel, je formulerai quatre séries d'observations.

1) Kelsen, on le sait, oppose de manière très tranchée, mais bien obscure, le monde des faits, du Sein, et le monde des normes, du Sollen, du devoir ou devoir-être, - - deux mondes mystérieux et irréductibles. En fait, le chef de l'École normativiste a reconnu lui-même n'avoir guère approfondi cette opposition Sein-Sollen reprise de Kant 
mais dont il n’a jamais dépassé une approche purement impressionniste :

La différence entre Sein et Sollen, être et devoir ou devoir-être, écritil, ne peut pas être expliquée davantage. Elle est donnée à notre conscience de façon immédiate. Personne ne peut nier que l'assertion que ceci ou cela est - c'est l'assertion qui décrit un fait positif - est essentiellement différente de la proposition que quelque chose doit être - c'est l'assertion qui décrit une norme; et personne ne peut nier que, du fait que quelque chose est, il ne peut pas suivre que quelque chose doive être, non plus qu'inversement de ce que quelque chose doit être, il ne peut pas suivre que quelque chose est ${ }^{5}$.

Le maître autrichien n'a pas su apercevoir clairement que les catégories modales impliquées dans les normes sont des catégories logiques, sans référent dans le monde extérieur : la possibilité, la nécessité, l'impossibilité ne sont pas des données d'observation, des éléments du tableau que le monde offre à notre regard. Ce sont, comme l'avait déjà bien vu Aristote, des notions produites de toutes pièces et mises en œuvre par l'esprit humain pour les besoins spécifiques de ses propres démarches et qu'il surajoute - qu'il surimpose - ainsi aux choses du monde. Autrement dit, et pour employer la terminologie kantienne, il s'agit de "catégories a priori». Et c'est précisément ce que vise essentiellement à exprimer l'opposition du Sein et du Sollen: il n'y a pas, dans le monde observable, de la possibilité ou de la nécessité comme il y a des arbres ou des étoiles; on ne peut donc tirer des normes à partir de la simple observation de la réalité : ce sont des construits de notre esprit qui s'articulent, dans leur principe même, autour de purs produits de notre logos, de pures entités logiques. Il n'y a là rien de très mystérieux.

2) Kelsen n'a pas vu non plus que les règles de conduite ne sont pas les seules à s'articuler autour des catégories modales : c'est le cas de toutes les règles, y compris les règles ou lois scientifiques élaborées par le savant. Au contraire, Kelsen oppose constamment les règles de conduite indiquant "si A est, B doit être, peut être ou ne peut pas être » et les lois scientifiques qui indiqueraient, elles, "si A est, B est ». C'est là une conception radicalement fausse, bien que couramment répandue. Il y a, sans doute, des différences d'espèce tout à fait irréductibles entre les règles scientifiques et les règles de conduite, mais elles appartiennent les unes et les autres, non moins indubitablement, au même genre, au genre règle: les lois scientifiques sont elles aussi des outils ayant pour vocation essentielle de donner la mesure du possible, plus précisément d'indiquer que «dans telles circonstances, tel type de phénomène doit se produire, ou ne peut 
pas se produire, ou peut se produire ou a tant de chances de se produire $^{6} »$. Ce n'est pas par hasard qu'on a été amené à les qualifier de « lois », encore que cette référence aux règles juridiques, inspirée par les arrière-plans métaphysiques et religieux qui ont historiquement présidé aux premières conceptions de la science ${ }^{7}$, ne soit pas dépourvue d'ambiguïté. En effet, ces règles n'ont évidemment pas pour vocation spécifique de diriger la conduite humaine (et encore moins de diriger la conduite de la nature, de la gouverner, ce qui n'a strictement aucun sens); elles n'indiquent pas les marges du possible de notre agir pour que nous ajustions nos faits et gestes sur elles; ce ne sont pas des outils de direction des conduites visant à encadrer la volonté humaine dans ses accomplissements, mais des outils de repérage face aux flux entremêlés d'événements que le monde nous donne à voir : leur vocation est de nous servir à nous repérer dans les productions du monde, à maîtriser mentalement le dédale des choses que la réalité fait défiler sous nos yeux, en bref à éclairer notre intelligence en lui signalant les degrés de possibilité qu'ont tel ou tel type de choses de se réaliser dans telles ou telles circonstances. Mais elles n'en restent pas moins, comme les règles de conduite, des construits de l'esprit, des outils mentaux ou artefacts intelligibles créés par lui pour ses propres démarches, sans doute en s'appuyant dans cette création sur des données d'observation, mais sans que ces règles soient pour autant elles-mêmes de simples observations, de simples constats ou tableaux du monde susceptibles d'être dits vrais ou faux, puisqu'elles s'articulent elles aussi autour de catégories modales sans référent dans le monde ${ }^{8}$. Elles sont d'autant moins susceptibles d'être dites vraies ou fausses qu'à l'instar des règles de conduite elles ne se réduisent pas à du logos, à de simples contenus de pensée assertés, mais correspondent à des contenus de pensée

6. Le fait, précisément que les lois scientifiques sont d'authentiques règles a inspiré à Galilée sa fameuse formule: "Le livre de la nature est écrit en langage mathématique » (Le messager céleste, 1610). En réalité, ce n'est pas la nature qui a une expression mathématique, mais les lois théoriques construites par la science, en tant même que règles s'articulant à l'instar de toutes les autres règles sur les catégories modales, lesquelles ne sont rien d'autre qu'une quantification sous forme de pourcentages - du possible. L'astrophysicien Laurent Notale observe pertinemment en ce sens: "La question que se posait Einstein et tant d'autres physiciens: "Comment se fait-il que les mathématiques puissent servir à décrire le monde ?» est peut-être une fausse question. Seule l'existence de lois pose un problème... Une fois celle-ci admise, leur expression trouve une forme naturellement compactée dans le langage mathématique " (La relativité dans tous ses états, Hachette, 1998, p. 29). Mais ce langage est aussi celui du droit et, plus généralement, de l'éthique, car il est de l'essence même des règles de mettre en œuvre une mathématique du possible.

7. Cf. mon étude «La science et le problème de la liberté humaine » publiée dans cette revue, vol. $27 \mathrm{n}^{\circ} 2$, automne 2000, p. $408 \mathrm{~s}$.

8. Voir mon étude déjà citée, Rev. dr. publ., 1992, p. 1036 s. 
réifiés, constitués en choses, en instruments ou appareils mentaux destinés à des utilisations spécifiques et donnant lieu, si l'on peut dire, à des manipulations de l'esprit : il s'agit non pas d'assertions, mais de formules, d'espèces de clefs ou grilles de lecture qui, appliquées mentalement sur le livre du monde, visent à permettre de pré-voir l'à-venir, ou de reconstituer le fil d'événements passés, ou bien encore de provoquer délibérément la production de telle ou telle chose en créant les circonstances adéquates qu'elles indiquent. Ce sont ces prévisions de la réalité à venir ou ces rétrospections de la réalité passée effectuées par leur truchement qui sont de nature à être vraies ou fausses en fonction des données expérimentales. Au contraire, et comme il en va pour tous les outils, y compris ceux du monde sensible, on ne peut à proprement parler tenir ces règles ellesmêmes pour vraies ou fausses, mais seulement pour bonnes ou mauvaises en fonction de leur valeur pragmatique, de leur utilité, de la qualité des services qu'elles rendent en pratique, c'est-à-dire selon qu'elles permettent plus ou moins efficacement de se repérer, selon le degré d'exactitude et de précision des prévisions et rétrospections que procure leur usage.

Précisément, c'est sous l'inspiration de vues logicistes réductrices méconnaissant la chosité des lois scientifiques, les ramenant aux seuls contenus de pensée dont elles sont faites et les assimilant à de pures assertions, qu'en logique normative ou déontique on qualifie couramment les catégories modales à l'œuvre dans le cadre de ces lois de "modalités aléthiques" par opposition aux "modalités déontiques " des règles de conduite: la nécessité, l'impossibilité et la possibilité ne seraient ainsi pas exactement de la même nature que l'obligation, l'interdiction ou la permission, même si l'on reconnaît une certaine " analogie » entre les unes et les autres. En réalité, il s'agit toujours des mêmes catégories du possible fonctionnant simplement dans des contextes instrumentaux différents, pour des usages différents, cette instrumentalisation leur imprimant une coloration particulière qui ne leur est pas inhérente et ne tient pas à leur essence propre. L'obligation n'est jamais que la nécessité de faire, l'interdiction l'impossibilité de faire, et la permission la possibilité de faire envisagées dans le cadre d'une direction de la conduite humaine: il n'y a pas de différence au niveau des catégories modales ellesmêmes en cause par rapport à la nécessité, l'impossibilité ou la possibilité de se produire des lois scientifiques. Au demeurant, sur un plan terminologique, il serait plus approprié d'appeler, non pas " aléthiques ", mais " ontiques » les modalités du possible lorsqu'elles sont utilisées dans l'ordre des faits, des étants du monde, ces modalités étant dites « déontiques » lorsqu'il en est fait usage dans l'ordre des à-faire des hommes. 
3) En ramenant à la suite de Kant les règles ou normes de conduite à l'idée de Sollen, en alléguant que "la norme est un "devoir-être» (Sollen $)^{9}$ ", Kelsen cherche à exprimer confusément que ces règles visent à diriger la conduite de ceux à qui elles sont adressées; mais au lieu de parler de "devoir » ou "devoir-être ", il aurait été mieux inspiré de parler de " pouvoir faire » : c'est l'idée générique du possible, de la possibilité d'agir qui caractérise le plus exactement ces règles (comme le révèle lumineusement la langue française qui place les règles juridiques sous l'appellation «Droit " évoquant l'idée du possible juridique). En utilisant le terme Sollen suggéré par l'objectif directif des règles de conduite pour caractériser l'être même de ces règles, leur consistance, Kelsen a été amené à croire que toutes les règles de conduite n'articulent que des obligations, des Sollen, et à éliminer laborieusement la catégorie modale des permissions devenue difficilement compréhensible dans son mode de pensée : comment, en effet, une norme pourrait-elle constituer un Sollen ou devoir permissif ? Finalement, les trois fonctions normatives, obligation, interdiction, permission, se ramèneraient à une seule véritable: l'obligation. C'est la seule fonction normative évoquée dans la première édition de la Théorie pure du droit. Dans la seconde édition, puis dans la Théorie générale des normes, Kelsen mentionne aussi l'interdiction et la permission, mais s'évertue à démontrer qu'elles ne font en réalité qu'un avec l'obligation.

Ainsi, l'interdiction est présentée simplement comme une variété d'obligation ou obligation de s'abstenir d'agir exactement de la même essence que l'obligation d'agir elle-même. Ce ne sont pas là, expose-t-il, "deux fonctions différentes, c'est au contraire une seule et même fonction relative à des comportements différents: une action et l'abstention d'une action ${ }^{10}{ }^{\prime}$. Et comme il s'agit d'actes contraires, les deux fonctions sont en définitive interchangeables ou réversibles à ses yeux : il revient au même d'interdire une action ou d'imposer l'obligation de s'en abstenir, d'obliger à accomplir une action ou d'interdire de s'en abstenir ${ }^{11}$.

Toute cette manière de raisonner est très superficielle, empreinte de paralogisme et passe en vérité à côté de l'essentiel. Bien sûr, lorsqu'un comportement est interdit, cela implique par hypothèse même — c'est là pure tautologie - qu'on ne peut pas se comporter de telle manière, qu'on doit ne pas se comporter ainsi; même formulée dans ces derniers termes, une norme articule une

9. Kelsen, op. cit., p. 7.

10. Théorie générale des normes, traduction Olivier Beaud et Fabrice Malkani, P.U.F., 1996, p. 125.

11. "Toute interdiction d'un comportement est le commandement de l'abstention de ce comportement, tout commandement d'un comportement donné est l'interdiction de l'abstention de ce comportement (ibid.). 
interdiction et non une obligation d'agir, mais Kelsen assimile ce "ne pas se comporter ainsi », cette exclusion logique impliquée par l'interdiction et qui exprime le degré nul de la possibilité, à un donné de fait, à un certain comportement (une espèce de comportement négatif ou "abstention ») qui serait en tant que tel l'objet d'une obligation ${ }^{12}$. De même, lorsqu'un comportement est obligatoire, cela implique qu'on ne peut pas ne pas se comporter ainsi; même formulée dans ces termes, une norme articule toujours une obligation et non une interdiction d'agir, mais là encore Kelsen assimile ce "ne pas se comporter ainsi ", cette exclusion logique impliquée par l'obligation et qui exprime le degré nul de l'impossibilité, à un certain comportement ou abstention qui serait en tant que tel l'objet d'une interdiction. Kelsen n'a pas compris le sens et l'irréductibilité de l'interdiction et de l'obligation en tant que degrés extrêmes opposés sur l'échelle bipolaire du possible de l'agir humain: l'interdiction ou degré minimal excluant, pour celui ou ceux dont on veut diriger la conduite, toute possibilité de faire telle chose et l'obligation ou degré maximal excluant toute possibilité de ne pas (c'est-à-dire toute impossibilité de) la faire, avec au milieu le degré intermédiaire de la permission incluant la possibilité de faire ou de ne pas faire cette chose.

Précisément, s'agissant de la permission, elle se rattacherait elle-même à l'obligation selon Kelsen. En effet, explique-t-i $1^{13}$, quand une norme indique qu' " on peut », cela peut avoir deux sens. En premier lieu, cela peut vouloir dire qu'il y a déjà par ailleurs une réglementation prohibitive générale en vigueur à laquelle on veut apporter une exception ; cette norme apparemment permissive est donc en réalité un élément d'une réglementation prohibitive : au lieu de formuler, par exemple, la règle générale prohibitive «il est interdit à tous les commerçants d'ouvrir le dimanche ", puis la règle en forme permissive « les boulangers peuvent ouvrir le dimanche », le législateur aurait pu simplement énoncer la norme prohibitive «il est interdit à tous les commerçants, sauf les boulangers, d'ouvrir le dimanche». Raisonnement purement artificiel qui méconnaît qu'on peut précisément tirer de la norme ainsi formulée, et malgré l'allure apparemment prohibitive de sa lettre, à la fois que les

12. Ne pas faire quelque chose, ce n'est pas faire le contraire, comme le croit Kelsen qui a des idées bien confuses à propos des actes contraires : il met ainsi sur le même plan l'accomplissement d'une action et son non-accomplissement, ou abstention, et l'acte de dire la vérité et l'acte de mentir qui sont d'authentiques actes contraires. Mais justement, s'abstenir de dire la vérité, ce n'est pas dire un mensonge, et obliger à dire la vérité n'équivaut pas à interdire de dire des mensonges. Kelsen aurait dû méditer à ce propos une mise en garde de son maître à penser : dans son Essai pour introduire en philosophie le concept de grandeurs négatives (1763), Kant invite, en effet, à bien distinguer entre les « oppositions logiques » (par exemple l'opposition entre être en mouvement et être au repos, c'est-à-dire ne pas être en mouvement) et les «oppositions réelles » (par exemple l'opposition entre se lever et se coucher): si ne pas être au repos équivaut à être en mouvement, ne pas se coucher n'équivaut pas à se lever (voir mon étude déjà citée, Rev. dr. publ., 1992, p. 1025 s.)

13. Théorie pure du droit, p. 22. 
boulangers " peuvent» ouvrir le dimanche et que les autres commerçants « ne peuvent pas » en faire autant.

La norme indiquant qu' " on peut " peut signifier, en second lieu, selon Kelsen, que telle personne ou catégorie de personnes a le droit de faire telle chose : ici, dit-il, la norme a le sens de conférer un droit (Berechtigen) et pas simplement d'ouvrir une possibilité (Erlauben). Mais ce droit ne fait que refléter une obligation, c'est en quelque sorte une obligation exprimée à l'envers :

Lorsque dans les rapports entre $\mathrm{A}$ et $\mathrm{B}$, écrit-il, il est prescrit à $\mathrm{A}$ de supporter que $B$ se comporte d'une certaine façon, on dit qu'il est permis à $B$ (c'est-à-dire que $\mathrm{B}$ a le droit) de se comporter de cette façon. Et lorsqu'il est prescrit à $\mathrm{A}$ de prester à $\mathrm{B}$ tel ou tel objet, on dit qu'il est permis à $\mathrm{B}$ (c'est-à-dire qu'il a le droit) d'obtenir de A la prestation en question. Alors, dans le premier cas, la phrase «il est permis à $\mathrm{B}$ de se conduire de telle façon » a exactement la même signification que la phrase «il est ordonné à A de supporter que B se comporte de telle façon »; et dans le second cas, le contenu de la phrase «il est permis à $\mathrm{B}$ d'obtenir telle prestation de $A$ » est exactement l'équivalent de la phrase «il est prescrit à $\mathrm{A}$ d'effectuer telle prestation à $\mathrm{B}$ ». Le caractère permis de la conduite de B n'est que la réflexion du caractère prescrit de la conduite de A. Ce permettre n'est pas une fonction de l'ordre normatif qui serait distincte de celle de prescrire $^{14}$.

Là encore le raisonnement de Kelsen apparaît bien artificiel: il n'y a aucune différence entre "pouvoir » et "avoir le droit de » en tant que permissions ou possibilités de faire ou de ne pas faire reconnues par des règles de conduite, et spécialement par des normes juridiques. Simplement, dans certains cas, la réglementation juridique offre par ailleurs, explicitement ou implicitement, aux intéressés une garantie en imposant à d'autres catégories de personnes l'obligation de ne pas apporter d'entraves ou certaines entraves à l'exercice de leur droit d'agir ou de ne pas agir et en leur ouvrant des voies de recours pour faire respecter cette obligation : dans ces cas, le droit reconnu aux intéressés reste un élément distinct et foncièrement différent de ces autres éléments de la réglementation et ne saurait en aucune manière être présenté comme un simple «reflet » de ces autres éléments ${ }^{15}$. De même, dans d'autres cas, la réglementation juridique impose à certains l'obligation de fournir telles ou telles prestations à d'autres et reconnaît à ces derniers le droit d'exiger, notamment devant les tribunaux, le respect de cette obligation. On parle souvent, dans ces cas, de "droits à ", de "droits » aux prestations en question ou encore de "droits de créance ", droits que l'on présente couramment, à l'instar de Kelsen, comme le reflet des obligations des débiteurs de prestations et qui se distingueraient ainsi des autres droits. C'est là une vue erronée

14. Théorie pure du droit, p. 22.

15. D'autant, d'ailleurs, que les mêmes garanties peuvent être reconnues par la réglementation aux titulaires, non pas de permissions, mais d'obligations, en imposant aussi que des entraves ne soient pas apportées par autrui à l'accomplissement de ces obligations : on n'a jamais eu l'idée de parler à ce propos d'obligations-reflets d'obligations. 
induite d'une manière peu rigoureuse de s'exprimer : il n'y a pas, à proprement parler, de «droit » à telle prestation, de "droit» de créance; un droit est toujours une possibilité de faire ou de ne pas faire. En l'occurrence, les prétendus titulaires de "droits à » ou "droits de créance » sont plus exactement des «bénéficiaires » des prestations qui leur sont dues en vertu des obligations instituées à la charge des débiteurs. Le seul droit proprement dit que leur reconnaît la réglementation, c'est la possibilité d'exercer des voies de recours en cas d'inexécution de leurs obligations par les débiteurs des prestations; mais ce droit de recours - ce droit d'exiger — est évidemment là encore un élément de réglementation distinct et foncièrement différent desdites obligations, un élément à part entière qui ne se confond pas avec elles, qui ne se dissout pas en elles : il est dépourvu de sens de le présenter comme en étant un simple " reflet» — d'autant, d'ailleurs, qu'il n'est pas nécessairement prévu par les textes qui instituent ce type d'obligations.

Si Kelsen s'est ainsi fourvoyé dans toutes ces impasses, c'est encore une fois parce qu'il n'a pas du tout saisi que l'obligation, la permission et l'interdiction correspondent aux trois degrés de l'échelle logique du possible, trois degrés qu'il est vain de prétendre ramener à un seul. Le manque d'élaboration de sa pensée en la matière éclate, du reste, dans un contresens magistral développé par lui à propos de l'obligation elle-même : il a été, en effet, amené à soutenir que lorsqu'une norme juridique oblige à faire telle chose sans que soit prévue une sanction en cas d'inexécution de cette obligation, cette obligation n'en serait pas vraiment une et il n'y aurait pas véritablement de norme dans cette hypothèse ${ }^{16}$ : comme si la catégorie modale du devoir n'était pas parfaite par elle-même et avait besoin du secours de cet élément complètement étranger de la sanction! Le maître autrichien confond manifestement ici une catégorie logique avec des données psychologiques ${ }^{17}$.

4) Dans le dernier état de sa pensée, Kelsen a cru pouvoir ajouter d'autres catégories aux catégories classiques de l'obligation, de l'interdiction et de la permission. Dans la deuxième édition de la Théorie pure du droit, il disait déjà que les fonctions normatives consistaient non seulement à ordonner de faire ou de ne pas faire et à permettre, mais aussi à « habiliter» — tout en semblant dans certains passages considérer l'habiliter comme une variante du permettre $^{18}$. Dans sa Théorie générale des normes, il élargit encore le

16. Voir par exemple Théorie pure du droit, p. $158 \mathrm{~s}$.

17. Il est proche sur ce point des idées de Bentham (cf. infra) qu'il a pourtant prétendu rejeter catégoriquement (Théorie générale des normes, p. 90 s.).

18. Voir par exemple Théorie pure du droit, p. 6 s.: " des actes portent en intention sur la conduite d'autrui quand ils ont pour signification, soit d'ordonner (ou commander) cette conduite, soit également de la permettre, et en particulier de l'habiliter, c'est-à-dire de conférer à l'autre un certain pouvoir, en particulier le pouvoir de poser lui-même des normes [...] aussi bien que commander, une norme peut permettre, et en particulier donner pouvoir". 
nombre des fonctions normatives en mentionnant, à côté du "commandement " (obligation positive ou négative) et de la "permission ", «l'habilitation» et "l'abrogation " ${ }^{19}$. C'est encore une autre manifestation d'une vision bien confuse de la nature même des normes et des modalités déontiques qu'elles articulent. Qu'est-ce, en effet, que ces deux prétendues fonctions ainsi mises sur le même plan que les catégories modales?

Il y a habilitation, dit Kelsen, quand une norme confère à un individu le pouvoir de poser ou d'appliquer des normes, « appliquer des normes » signifiant pour lui requérir une sanction (comme il appartient, par exemple, dans une instance pénale au ministère public ou à un plaignant), prononcer une sanction (comme il revient couramment aux autorités juridictionnelles) ou exécuter la sanction prononcée (cette exécution pouvant incomber, par exemple, aux gendarmes, aux gardiens de prisons ou aux bourreaux $)^{20}$. Il est curieux de rassembler ainsi sous la même étiquette des hypothèses aussi disparates dont on discerne mal le dénominateur commun. Mais surtout, ces actes d'édiction ou d'application de normes, que la réglementation «habiliterait » à accomplir, sont en vérité des actes humains comme les autres et des actes que la réglementation encadre exactement comme les autres en indiquant, selon les cas, ce qui peut, ce qui ne peut pas ou ce qui doit être accompli par les intéressés : il n'y a pas une modalité " habilitation " à côté de ces trois modalités. Kelsen reconnaît lui-même que ces actes « habilités " peuvent être, selon la réglementation, obligatoires pour les intéressés, mais ne le sont pas nécessairement; ils peuvent être seulement permis, ils peuvent aussi être interdits ${ }^{21}$ : c'est bien que la prétendue « habilitation » n'est pas une fonction propre à côté de l'obligation, de la permission et de l'interdiction. En fait, ce que Kelsen appelle «habilitation" parait se ramener tout simplement à ce qu'on nomme plus couramment dans la théorie juridique - et spécialement dans la théorie du droit public — «compétence ", c'est-à-dire le pouvoir agir reconnu aux différentes catégories d'acteurs juridiques - et spécialement aux autorités publiques - tel qu'il résulte des différentes marges qui leur sont fixées par la réglementation.

L'abrogation n'est pas, elle non plus, une fonction normative ou déontique. Kelsen a été victime ici du manque de pertinence et d'approfondissement de ses vues en matière d'actes juridiques ${ }^{22}$. Pour lui, et bien qu'il le qualifie d'acte de volonté, un acte d'édiction de normes juridiques, c'est simplement

19. Théorie générale des normes, p. $125 \mathrm{~s}$.

20. Théorie générale des normes, p. 137.

21. Théorie générale des normes, p. $134 \mathrm{~s}$.

22. Voir à ce propos mes contributions sur «l'acte juridique à travers la pensée de Charles Eisenmann" (in La pensée de Charles Eisenmann, sous ma direction, Economica, 1986, p. 47 s.) et "Le locutoire et l'illocutoire dans les énonciations relatives aux normes juridiques" (Revue de métaphysique et de morale, 1990, p. 400 s.). 
un acte de dire des normes juridiques, une énonciation de normes juridiques. Il n'a pas su apercevoir l'épaisseur pragmatique de cet acte de parole, sa nature (sa «valeur illocutoire" comme diraient les spécialistes de la théorie des actes de langage) d'acte d'autorité consistant, non pas seulement à énoncer des normes, mais à les " poser » ou les "établir ", c'est-à-dire à les mettre en vigueur, à les rendre applicables, à les mettre autoritairement en service, en activité, dans un certain espace social qu'elles sont ainsi appelées à régir. Cette « force » des normes juridiques découlant de l'acte même de mise en vigueur accompli par le locuteur — par le législateur — dans l'exercice de ses fonctions sociales de commandement, Kelsen l'a assimilée à leur «validité ", cette dernière signifiant pour lui tout à la fois que ces normes sont «valables » (gilt), qu'elles ont été régulièrement édictées, en conformité avec les normes supérieures régissant cette édiction, et que conséquemment elles sont obligatoires (verbindlich), en vigueur ${ }^{23}$ : de sorte que cette "validité » ne provient pas, à ses yeux, de la nature même de l'acte d'édiction, mais uniquement des normes supérieures en conformité desquelles il a été accompli.

N'ayant pas su rendre compte des actes de mise en vigueur des normes juridiques, Kelsen ne pouvait qu'avoir des idées brouillées de ces autres actes juridiques d'autorité que sont les actes de mise hors vigueur, hors service, temporairement ou définitivement, et les actes de modification de la réglementation antérieurement mise en vigueur qui sont des actes contractant à la fois une mise hors vigueur des dispositions modifiées dans leur teneur initiale et une mise en vigueur d'un dispositif nouveau dont la teneur résulte de la modification apportée : exercer une fonction sociale de commandement, ce n'est pas simplement faire entrer en vigueur des règles, mais plus exactement moduler à l'adresse des dirigés le tissu de règles en vigueur qu'ils ont à observer, à mettre en pratique dans leurs conduites. Concevant l'acte de mise en vigueur comme un acte purement énonciateur d'une norme, Kelsen a été amené à se représenter corrélativement l'acte de mise hors vigueur également comme un acte énonçant une norme, mais une norme "négative »: il s'agirait, prétend-il, d'un "acte de législation négative", le législateur faisant office en l'occurrence de "législateur négatif ${ }^{24}$. La norme négative ainsi émise, statuant "non pas un devoir-être, mais un non-devoir-être ", aurait par là une fonction originale qui ne serait ni d'imposer, ni d'interdire, ni de permettre un certain comportement, mais d'abroger une autre norme, de supprimer sa validité $^{25}$. Kelsen n'a guère été troublé par l'atypisme de cette prétendue variété de normes, qu'il a pourtant lui-même souligné : qu'est-ce que ces règles

23. Théorie pure du droit, p. 10 s. et p. 255. À différentes reprises, Kelsen assimile expressément «validité » et « vigueur» (par exemple p. 11: « une norme qui vaut, qui est en vigueur...»; ou p. 14 : «la norme n'acquiert validité, elle n'entre en vigueur $[. .] »$.$) .$

24. Kelsen, Théorie générale du droit et de l'État, traduction Béatrice Laroche et Valérie Faure, Bruylant-L.G.D.J., 1997, p. 317. Ces idées ont été reprises en particulier par Charles Eisenmann (Cours de droit administratif, tome 2, L.G.D.J., 1983, p. 352 et 417 s.).

25. Théorie générale des normes, p. $139 \mathrm{~s}$. 
de conduite qui ne viseraient pas des conduites humaines mais des normes et qui seraient par suite insusceptibles d'être observées ou appliquées, qui ne pourraient non plus être violées, qui seraient du même coup dépourvues par ellesmêmes de "validité », de force obligatoire ${ }^{26}$ ?

\section{Les courants négationnistes dans la pensée juridique et éthique}

La théorie de la science a toujours été réfractaire à apercevoir dans les lois scientifiques des lois ou règles authentiques - comme si les règles étaient seulement des règles de conduite, des règles pratiques encadrant la gouverne des hommes dans la production de leurs faits et gestes, et ne pouvaient pas être aussi des règles théoriques encadrant leur lanterne face aux productions du monde dont ils sont spectateurs. L'on a tendu jusqu'ici à occulter complètement les catégories modales du possible que ces «lois de la nature »comme on les appelle encore couramment - articulent, et l'on se représente généralement ces dernières comme des descriptions du monde susceptibles d'être dites vraies ou fausses; plus précisément, une loi scientifique serait simplement une description des conditions de survenance d'un type de phénomène dans toute la série de ses apparitions au monde, passées et futures: "Chaque fois que telles circonstances se réalisent, tel type de phénomène se produit. » Ces vues générales - universelles — relatives à l'ensemble des manifestations historiques d'un phénomène seraient inférées à partir des cas d'espèce expérimentés par le savant : elles seraient induites de ces observations particulières, cette opération logique d'induction revêtant en l'occurrence un caractère problématique (le fameux "problème de l'induction scientifique ") puisqu'on ne peut jamais être sûr qu'une proposition générale inférée de quelques propositions particulières vraies soit elle-même vraie. Les énonciations scientifiques correspondraient ainsi à d'audacieux pronostics ou paris sur l'avenir, rien ne permettant d'affirmer avec certitude que tel phénomène dont on a observé la survenance dans certaines circonstances se produira toujours, invariablement, dans les mêmes circonstances; elles n'auraient jamais que la valeur de pures hypothèses ou conjectures, toujours susceptibles d'être infirmées — «falsifiées ", comme dit Karl Popper — par l'expérience.

Cette conception couramment répandue des lois scientifiques a exercé une profonde influence sur les théoriciens du droit et de l'éthique : soit que

26. Ayant défini la validité comme le "mode spécifique d'existence » des normes, Kelsen a dû reconnaître, par un tour particulièrement audacieux de haute voltige intellectuelle, une certaine validité - une sorte de validité fugace ou instantanée — à la norme abrogatoire : "elle ne peut pas être valide par elle-même, explique-t-il (Théorie générale des normes, p. 140), elle peut être valide seulement en relation avec une autre norme, la norme dont elle supprime la validité. Les normes abrogatoires sont en ce sens des normes non indépendantes » : assez bizarrement, on le voit, la validité ici ne proviendrait plus de la norme supérieure à laquelle l'acte d'édiction de la norme abrogatoire serait conforme, mais de la norme abrogée elle-même, norme dont la norme abrogatoire dans le même instant emprunterait et supprimerait la validité. En quelque sorte, la norme abrogatoire s'autodétruirait elle-même en supprimant «l'existence » de la norme abrogée dont elle tirerait elle-même sa propre «existence »... 
ces théoriciens l'aient reprise pour opposer radicalement les règles juridiques et éthiques aux lois scientifiques, simples descriptifs déconnectés de toute catégorie modale, - c'est le cas par exemple, on l'a vu plus haut, de Kelsen; soit - et c'est ce qui nous intéresse ici - que ces théoriciens aient succombé à la folle tentation de placer le domaine du droit et de l'éthique sous le label flamboyant de la science et de l'expérience et qu'ils aient été ainsi amenés à concevoir les règles juridiques et, plus largement, éthiques sur ce modèle généralement admis des lois scientifiques, en prétendant dénier toute consistance véritable aux modalités déontiques, en développant à leur propos des thèses négationnistes. Les notions de "droit" et "d'obligation " dans le domaine des règles pratiques seraient des notions archaïques, fictives, parfaitement superflues et mystificatrices dont il conviendrait — dans un souci de réalisme - de débarrasser la théorie juridique et éthique. Ce courant négationniste correspond de fait à une tendance tenace : il s'est manifesté à bien des reprises, à des époques et sous des cieux différents, à travers des représentants venant d'horizons de pensée fort divers. Il n'est pas question dans ces quelques pages d'en présenter une étude exhaustive et approfondie, mais d'en faire un rapide survol à travers ses illustrations les plus saillantes.

1) Dans la pensée juridique française contemporaine, Michel Villey est incontestablement la figure de proue du courant négationniste ${ }^{27}$. Ses convictions jusnaturalistes l'ont conduit, en effet, à envisager le droit comme une démarche de pure connaissance visant non pas à " prescrire une conduite» — ce qui serait du ressort de la morale — mais à "indiquer une réalité ", plus précisément à indiquer "ce qui est à chacun », la « juste part » qui lui revient et qui se trouve inscrite dans la nature des choses : c'est ce que traduirait la fameuse formule romaine "sum cuique tribuere". Les notions d'obligation, d'interdiction et de permission utilisées par le législateur et par la théorie juridique seraient des notions superfétatoires, illusoires, des espèces de trompe-l'œil inspirés par une confusion du droit avec la morale qui remonterait à la pensée biblique. Débarrassé de ces scories, le droit - jus - dans son essence véritable « dit ce qui peut être aperçu du juste dans les rapports sociaux, comme le poète décrit le beau perçu dans les choses ". Cette nature fondamentalement descriptive serait attestée par l'usage même, très fréquent, de l'indicatif — « mode le mieux approprié » — dans les énoncés juridiques,

27. Voir notamment son précis Philosophie du droit, tome 1, Dalloz, $1975, \mathrm{n}^{\circ} 35$ s., et surtout son article «De l'indicatif dans le droit ", Archives de philosophie du droit, 1974-19, p. 33 s. (dont sont extraits les fragments cités au texte). On retrouve un négationnisme comparable à l'étranger dans les conceptions partageant la même approche "cognitiviste » des valeurs; c'est en particulier le cas de l'axiologie phénoménologique développée dans le sillage d'Edmund Husserl et Adolf Reinach et selon laquelle les normes ne seraient que l'expression de "valeurs » objectivement constatées : «on doit faire ceci » serait l'équivalent de «il est bon de faire ceci », "faire ceci est naturellement doté d'une valeur positive saisissable par l'intuition". 
particulièrement en droit romain : "Le discours juridique romain n'est pas déontique. Il n'y est pas question de ce qu'on doit ou ne doit pas faire (déontologie) [...] On ne s'y occupe point de nos conduites [...] Ce discours vise à indiquer ce qui est à chacun - indicatif. " La même observation vaudrait pour le droit moderne, et par exemple pour le Code civil français lui aussi «écrit à l'indicatif et à l'indicatif présent ». Sans doute, reconnaît Villey, « le futur se rencontre aussi; et nous constatons qu'il est même la forme ordinaire dans le Code pénal ». Mais ces descriptions au futur s'expliqueraient par le fait que le législateur ne parvient pas toujours à discerner clairement le juste immanent à la réalité et qu'il est ainsi appelé à inventer pour suppléer l'insuffisance de ses observations : «Il s'agit alors de juste positif, c'est-à-dire créé par la loi, et qui par définition même n'existera qu'une fois la loi promulguée. " Mais, souligne Villey, «quand bien même l'office du juriste n'est pas purement spéculatif [...], reste que le droit proprement dit n'entretient pas de relation immédiate avec la praxis; il ne vise pas à commander les conduites humaines, il dit ce qui est ou qui sera.»

S'inscrivant dans cette visée de simple reconnaissance de ce qui est ou revient à chacun, les "droits" correspondraient, non pas à de prétendues permissions ou pouvoir agir, mais à une attribution de choses, biens corporels ou avantages; de même, les " obligations " et les «interdictions " correspondraient, non pas à des devoir faire ou ne pas faire, mais à une attribution de choses ou valeurs "négatives» telles que des dettes, des désagréments ou des désavantages. C'est ainsi qu'il faudrait interpréter, par exemple, les dispositions du Code pénal : "Le droit pénal n'a pas pour fonction d'interdire, quoi que certains prétendent, l'homicide, le vol ou l'avortement; ces défenses relèvent de la morale; un jury ou le Code pénal répartissent les peines, à chacun la peine qui lui revient ${ }^{28}$. " Les normes du droit pénal se réduiraient ainsi à des espèces de formules de tarification: "Voici ce que coûte le vol ou le meurtre", "Voici ce qui revient à celui qui vole ou qui tue.»

2) Ce sont aussi des vues négationnistes que l'on voit s'exprimer, mais sous des inspirations radicalement différentes et même opposées, dans le mouvement réaliste qui a vu le jour à partir de la fin du XIX ${ }^{e}$ siècle dans la pensée juridique américaine. Aux antipodes de toute conviction métaphysique et se défiant même du "théorique » en général, se voulant - dans la lignée du pragmatisme de Charles Sanders Peirce, William James et John Dewey — résolument réalistes et

28. Philosophie du droit, tome $1, \mathrm{n}^{\circ}$ 40. Cpr. Philippe Jestaz qui juge à cet égard « ringarde " «l'idée que la norme serait une règle de conduite " (note in Archives de philosophie du droit, 1998-42, p. 418). 
démystificateurs, les représentants de ce mouvement ont été amenés, dans leur souci même de réalisme, à développer à propos du droit et de sa théorie des idées réductrices assez frustes. Pour eux la théorie du droit en tant que «science » — et conformément à la conception dominante du travail et des lois ou propositions scientifiques - doit être uniquement axée sur des faits et s'efforcer de formuler à leur propos des prévisions fiables. En l'occurrence, les faits dont la science juridique a à s'occuper, ce sont les décisions judiciaires : c'est qu'en effet, pour la realistic jurisprudence, «le droit, c'est ce que les juges disent être le droit ${ }^{29}{ }^{2}$; toute l'expérience juridique tourne autour du juge et est réduite à une expérience judiciaire $^{30}$. Les règles posées par le législateur n'auraient en tant que telles aucune importance véritable d'un point de vue "réaliste » : ces règles dépendent, en effet, de ce que le juge en fera, de sorte que le droit réel - le droit à l'œuvre dans la réalité et tel que devrait l'exposer une "science juridique » authentique - , c'est uniquement ce que le juge sera amené à décider dans les cas d'espèce dont il aura à connaître. Herbert Hart résume ainsi l'esprit général du réalisme américain : «Parler de règles constitue un mythe qui masque la réalité, à savoir que le droit consiste simplement dans les décisions des tribunaux et la prédiction qu'on peut en faire ${ }^{31}$.» Telle est bien l'emblématique profession de foi exprimée par Holmes : «Les prédictions de ce que feront les tribunaux, et rien d'autre de plus prétentieux, sont ce que j'entends par droit ${ }^{32}$. » Les règles édictées par le législateur sont conçues tout au plus comme des instruments destinés à permettre d'effectuer ces prédictions, encore qu'il faille tenir compte par ailleurs de bien des facteurs circonstanciels susceptibles d'influer sur les décisions judiciaires. C'est ce que souligne, par exemple, Karl Llewellyn : "Les règles sont importantes dans la mesure où elles nous aident à prédire ce que les juges vont faire. C'est en cela que réside leur importance, à part le fait qu'elles constituent de beaux jouets ${ }^{33}$.»

En définitive, on le voit, au nom d'une prétendue exposition réaliste et scientifique du droit, la réglementation juridique et les modalités déontiques qu'elles impliquent se trouvent complètement dénaturées :

29. Françoise Michaut, L'école de la sociological jurisprudence et le monvement réaliste américain, Université de Lille III, 1985, p. 70.

30. Il est vrai que beaucoup des réalistes américains ont été eux-mêmes des juges, notamment à la Cour suprême (par exemple Holmes, Cardozo ou Douglas).

31. Herbert Hart, Le concept de droit, traduction Michel Van de Kerchove, Facultés universitaires Saint-Louis, Bruxelles, 1976, p. 169.

32. Oliver Wendell Holmes, "The Path of the Law", Harvard Law Review, 1897, vol. 10, p. 466.

33. Karl Llewellyn, The Bramble Bush. On our Law and its Study, Oceana Publications, New-York, 2 édition, 1951, p. 14. 
Les droits et obligations élémentaires dont s'occupe la science du droit ne sont pas autre chose que des prédictions [...] ce qu'on appelle obligation juridique n'est pas autre chose que la prédiction de tel ou tel mal qui sera infligé à un homme en vertu du jugement du tribunal si cet homme fait ou omet de faire quelque chose ; il en va de même pour ce qui est du droit $^{34}$.

3) Dans cette manière de vider de leur signification propre les catégories modales à l'œuvre dans les règles juridiques, les réalistes américains s'inscrivent eux-mêmes dans le sillage des idées développées par Jeremy Bentham entre la fin du XVIII ${ }^{\mathrm{e}}$ siècle et le début du XIX ${ }^{\mathrm{e}}$. Le fondateur de la morale utilitariste a, en effet, développé dans différents écrits, et en particulier dans ceux publiés sous le titre $O f$ Ontology $y^{35}$, une théorie des fictions inspirée pour une grande part par une réflexion sur le droit. Selon Bentham, notre pratique langagière articule inévitablement ce qu'il appelle des "entités fictives ", des entités qui n'ont pas de réalité, pas de référent observable dans le monde, mais qui correspondent simplement à nos manières de parler, de nous exprimer, et " dont on ne pourra jamais se passer aussi longtemps que le langage sera en usage parmi les êtres humains ${ }^{36}$ ". Bentham cite, par exemple, "des mots tels que Relation, Situation, Faculté, Pouvoir et d'autres du même genre ». Plus précisément, " une entité fictive est une entité à laquelle on n'entend pas attribuer en vérité et en réalité l'existence, quoique, par la forme grammaticale du discours que l'on emploie lorsqu'on parle d'elle, on la lui attribue ${ }^{37}$ » : on parle, en effet, de ces êtres purement langagiers ou verbaux de la même façon que l'on parle d'entités réelles, "comme si » elles étaient des entités réelles, en les mettant déjà sur le même plan que ces dernières par le fait même de les désigner aussi par un substantif, ce qui paraît renvoyer à une substance réelle. Ainsi, "c'est au langage, et au langage seul, que les entités fictives doivent leur existence, leur impossible et néanmoins indispensable existence ${ }^{38}$ ". Mais, en pratique, on a plus ou moins tendance à perdre de vue qu'il s'agit de fictions et à les hypostasier, à les concevoir comme des entités réelles, bien entendu illusoires, "fallacieuses »: on transforme ce qui est simple procédé langagier en affabulation, des mots en êtres fabuleux qui existeraient réellement. On dispose, toutefois, d'un antidote pour ne pas céder à cette

34. Oliver Wendell Holmes, op. cit.

35. Cet ouvrage, qui se compose de textes rédigés entre 1813 et 1821, a été édité avec d'autres textes annexes en version française aux Éditions du Seuil en 1997 (traduction et commentaires de Jean-Pierre Cléro et Christian Laval). C'est cette édition qui sera citée au texte.

36. De l'ontologie, p. 87.

37. Op. cit., p. 165 (voir aussi p. 87).

38. Op. cit., p. 85. 
pente : il est possible, en effet, de dissoudre une entité fictive en exprimant autrement ce qu'on veut exprimer à travers elle, en recourant à des paraphrases n'articulant d'elles que des entités réelles.

Bentham a relevé des entités fictives dans bien des secteurs de notre pratique discursive : la matière et la forme, la quantité, la qualité, le temps, le lieu, l'existence, sont ainsi cités comme des entités physiques fictives, fictivement abstraites des entités physiques réelles dont elles font partie; de même, «facultés-pouvoirs de l'esprit, dispositions : toutes ces entités sont irréelles. Elles ne sont toutes qu'autant d'entités fictives ${ }^{39}{ }$ ". Mais ce sont les entités fictives du droit qui ont principalement intéressé Bentham : la plupart des catégories utilisées dans ce domaine ont, pour lui, cette nature et appellent donc une salutaire déconstruction par la méthode de la paraphrase ${ }^{40}$. Et parmi elles, ce sont précisément les "droits» et les «obligations » qui ont surtout retenu son attention: il s'agit là, en effet, à ses yeux, de cas typiques de fictions n'ayant d'autre réalité que purement langagière. Ainsi, l'obligation serait uniquement une manière de dire que ne pas avoir tel comportement ou au contraire - dans le cas d'une obligation de ne pas faire - avoir tel comportement va entraîner une sanction, une peine ou un désagrément :

Une obligation (c'est-à-dire l'obligation de se conduire d'une certaine manière) est imposée à un homme, ou plutôt on dit qu'elle est imposée à un homme si, pour le cas où il manquerait de se conduire de cette manière, la douleur ou la perte de plaisir est envisagée comme ce dont il doit subir l'épreuve. Comme ce n'est pas le nom d'une entité réelle mais seulement celui d'une entité fictive, et comme cette dernière n'a pas de genre supérieur, cette entité n'est pas considérée comme susceptible de définition sous la forme ordinaire, per genus et differentiam, mais plutôt comme redevable d'une exposition par la voie de la paraphrase [...]. On peut bâtir sur l'exposition que nous avons donnée du terme obligation, qui servira ainsi de base, les autres expositions des droits, quasi-droits ou avantages analogues aux droits $^{41}$.

39. Op. cit., p. 173.

40. "Pouvoir, droit, prohibition, devoir, obligation, charge, immunité, exemption, privilège, propriété, sécurité, liberté, ainsi qu'une multitude d'autres termes que l'on pourrait citer, sont tous autant d'entités fictives qui peuvent être considérées, pour parler communément, comme des créations et des instruments du droit dans des circonstances données. Il n'est pas une opération que le droit n'accomplisse sans que l'on ne voie la création et l'utilisation d'une façon ou d'une autre de ses productions imaginaires. Il est clair que tout ceci n'est qu'une pure œuvre de fantaisie, une sorte d'allégorie, une énigme dont la solution ne peut être donnée qu'en retraçant l'histoire des opérations qu'effectue le droit en relation à certaines entités réelles [...]. Ces dénominations fantastiques constituent une sorte de papier-monnaie : si nous savons à tout instant comment les échanger et comment obtenir à leur place de la monnaie de bon aloi, tout est pour le mieux. Si nous ne le pouvons pas, nous sommes trompés, et au lieu d'être les maîtres d'une connaissance réelle dont nous entendions nous pourvoir par leur moyen, nous n'avons alors que sophistique et non-sens » (Of Laws in General, cité dans De l'ontologie, p. 221 s.).

41. Essay on Logic, cité dans De l'ontologie, p. 213 s. 
Dire que quelqu'un a le «droit» de faire telle chose se ramènerait donc uniquement à indiquer que, s'il fait cette chose, il n'encourra aucune sanction, aucune douleur, il pourra goûter au plaisir d'agir sans crainte $^{42}$, autrui s'exposant par contre, le cas échéant, à des désagréments s'il essayait de l'empêcher d'agir.

En qualifiant ainsi de fictives les catégories modales élaborées par les règles juridiques et plus généralement éthiques, Bentham commet un contresens manifeste. Sans doute s'agit-il, on l'a vu, de catégories purement logiques sans référent dans le monde extérieur; sans doute aussi nos manières usuelles de parler peuvent induire en erreur si on les prend à la lettre, si on donne notamment le même sens à " avoir un droit (ou une obligation) » et à " avoir deux yeux » ou "avoir deux euros en poche». Mais ces notions ne sont pas fictives pour autant; ce ne sont pas des espèces de fantasmes suscités par notre pratique langagière et qu'une modification de celle-ci ferait s'évanouir. Peu importe qu'elles n'aient pas de référent dans le monde extérieur; elles interviennent au niveau des opérations de l'esprit lui-même et des outils construits et utilisés par lui, en particulier les règles de conduite: à ce niveau elles jouent en tant que telles, avec leur signification propre et irréductible à une autre, un rôle réel et nullement fictif, qui se traduit à travers les accomplissements observables des usagers de ces outils. Les choses du monde sensible ne sont pas les seules entités réelles; les produits de l'esprit, actes et objets mentaux, y compris les concepts ou catégories, sont aussi des réalités, comme l'a particulièrement souligné la phénoménologie husserlienne ou, plus récemment, Karl Popper avec ses entités du «Monde $3{ }^{43}$.

42. Pour Bentham, «la douleur peut avoir pour équivalent une perte de plaisir, et le plaisir de même peut avoir pour équivalent un évitement de la douleur» (ibid.).

43. Karl Popper, La quête inachevée, traduction Renée Bouveresse, Calmann-Lévy, 1981 , p. 262 s. Voir à ce propos mes observations dans "Le droit dans les esprits", in Controverses autour de l'ontologie du droit, sous ma direction, P.U.F., 1989, p. 42 s., et «Les règles juridiques en tant qu'objets mentaux ", Archiv für Rechts-und Sozialphilosophie, vol. 791993, Heft 3, p. 315 s.

En fait, la théorie des fictions de Bentham amalgame confusément deux choses très différentes. On a tendance en pratique, à travers nos façons mêmes de nous exprimer, à donner une réalité naturelle, indépendante de nous, à ce qui n'est que produit de notre esprit. Par exemple, nous inclinons facilement à hypostasier les classements que notre esprit opère à l'égard des choses du monde, à croire que celles-ci « se divisent » naturellement sous nos yeux en classes qui se donneraient ainsi à observer avec elles, qui feraient partie avec elles du mobilier du monde; de même, nous sommes facilement portés à projeter dans le monde lui-même les rapports ou relations que notre esprit établit entre les choses, à imaginer ainsi que le savant se contente d'observer les rapports qui «existeraient » entre les phénomènes ou encore qu'il ne ferait que découvrir les «lois de la nature " qui seraient présentes en tant que telles en face de lui. Mais une chose est d'observer cette tendance de l'esprit à s'occulter dans ses propres productions, à oublier qu'il en assume la paternité; une autre chose est de tenir les produits de l'esprit pour de simples fictions induites par le langage et qu'on pourrait dissiper en s'exprimant différemment. 
Ce négationnisme à l'égard des catégories modales revient en tout cas, là encore, à donner une vue complètement tronquée des règles éthiques, en en faisant non plus des instruments de direction des conduites, mais des instruments de persuasion, d'action sur les motivations - le législateur étant lui-même réduit au simple rôle « de distribuer la douleur, ou du moins la menace de la douleur ${ }^{44} »$. Cette vision, il est vrai, se situe parfaitement dans le prolongement des idées utilitaristes professées par Bentham et qui font du plaisir et de la douleur les seuls véritables moteurs des comportements humains. C'est à partir de là qu'il a construit sa théorie de l'éthique, - ce qu'il a appelé "déontologie » ${ }^{45}$.

4) La réduction des catégories modales à l'œuvre dans l'éthique et le droit à des fictions inhérentes à nos pratiques langagières; on les retrouve également dans les conceptions du positivisme logique qui a prospéré au cours de la première moitié du $\mathrm{XX}^{\mathrm{e}}$ siècle dans le prolongement du Cercle de Vienne. Ce courant philosophique repose, on le sait, sur le principe selon lequel - en dehors des énoncés analytiques à contenu purement tautologique — les seuls énoncés pourvus de sens sont ceux qui ont un contenu factuel, qui décrivent des faits empiriques et dont on peut contrôler expérimentalement s'ils sont vrais ou faux. Tous les autres énoncés sont considérés comme dénués de sens, non pas simplement faux mais absurdes, vides de contenu intelligible: ces énoncés métaphysiques correspondent à une espèce de fausse monnaie linguistique qui circule et abuse les esprits tant qu'on ne s'aperçoit pas qu'il ne s'agit que de pur papier linguistique ne valant rien, ne voulant rien dire. Dans cette perspective, les énoncés éthiques en général - les énoncés de valeur et non de fait empirique - et les énoncés de règles de conduite en particulier devraient se trouver condamnés à être rangés parmi ces énoncés métaphysiques. Mais le positivisme leur a reconnu une place à part dans la catégorie des énoncés non signifiants, ne disant rien sur le monde empirique: ils auraient, en effet, la nature de pures manifestations émotionnelles, d'expressions de sentiments intérieurs au locuteur.

Cette conception a abouti à dénier aux modalités déontiques leur identité propre et leur rôle spécifique : le « devoir » de l'adressataire d'une norme morale ou juridique se ramènerait en réalité simplement à

44. Gérard Ginestier, notice sur «Bentham » in Dictionnaire des philosophes, sous la direction de Denis Huisman, vol. 1, P.U.F., 1984, p. 267.

45. L'ouvrage de Bentham Introduction aux principes de morale et de législation (1789) s'ouvre par cette célèbre phrase emblématique: "La nature a placé l'humanité sous la gouverne de deux maîtres souverains, le plaisir et la douleur. C'est à eux, et à eux seuls, de nous indiquer ce que nous devons faire comme de déterminer ce que nous allons faire. Sont attachées à leur trône d'un côté les normes du bien et du mal et de l'autre la chaîne des causes et effets. » 
l'expression d'un désir, d'un désir pressant du locuteur émetteur. C'est ce qu'a soutenu en particulier le fondateur du positivisme logique, Moritz Schlick :

Je dois faire quelque chose ne signifie jamais rien d'autre que quelqu'un d'autre désire que je le fasse; et certes le désir d'autrui, qui m'est adressé, est désigné comme un devoir-être seulement quand cette personne est en mesure de conférer une certaine pression à ce désir, et donc de récompenser son accomplissement, ou punir son non-accomplissement, ou au moins d'avertir de toutes les conséquences naturelles de l'obéissance ou de la négligence ${ }^{46}$.

On doit souligner, au passage, une certaine contradiction dans le positivisme juridique, et notamment chez Schlick : d'un côté, en effet, on considère les énoncés de règles dans le domaine de l'éthique comme des non-sens, puisque sans contenu factuel; d'un autre côté, on admet que les lois scientifiques - conçues, selon les idées couramment reçues et par-delà les catégories modales qu'elles articulent elles aussi, comme des énoncés purement descriptifs à caractère général — ne sont pas, du fait même de cette généralité, des propositions susceptibles d'être dites vraies ou fausses (ce à quoi Popper objectera, d'ailleurs, qu'elles peuvent au moins être «falsifiées », infirmées par l'expérience), mais qu'elles ont du sens néanmoins en tant qu'instruments de production d'énoncés prédictifs apophantiques dans des cas particuliers, - ce qui revient bien à reconnaître qu'il s'agit d'outils mentaux spécifiques et non de véritables propositions descriptives. Le positivisme logique réserve ainsi à deux espèces d'outils mentaux appartenant au même genre - les règles pratiques ou éthiques et les règles théoriques ou scientifiques - deux traitements radicalement différents. Pourtant, ces deux sortes de règles, lorsqu'on les applique à des cas concrets (lorsqu'on leur subsume des cas concrets), donnent pareillement lieu à des énoncés qu'on peut dire à caractère prévisionnel ou projectif, indiquant ce que "prévoient » ces règles dans ces cas, c'est-à-dire ce qui va certainement ou éventuellement se réaliser ou ne pas se réaliser (pour les lois scientifiques), ce qui est forcément, ou au gré de l'agent concerné, à réaliser ou à ne pas réaliser (pour les règles éthiques); et ces énoncés sont pareillement susceptibles de contrôle expérimental, de confrontation à la réalité. Simplement, ce contrôle aboutit à établir la vérité ou la fausseté de l'énoncé projectif émis sur la base de la loi scientifique, et indirectement à donner la mesure de

46. Moritz Schlick, Fragen der Ethik, Springer, Vienne, 1930, p. 81. Voir: Kelsen, Théorie générale des normes, p. 92 s.; Christian Bonnet, "Le positivisme éthique de Schlick", Les Études philosophiques, 2001-3, p. 371 s. On retrouve des vues similaires exposées chez d'autres représentants du positivisme juridique, par exemple Alfred Jules Ayer pour qui les normes éthiques servent pareillement à la fois "à exprimer des sentiments " et "à susciter des sentiments, et ainsi à stimuler l'action" (Langage, Vérité et Logique, 1936, traduction Joseph Ohanna, Flammarion, 1956, p. 152). 
la validité ou de la valeur pragmatique de cette dernière, c'est-à-dire de son efficacité en tant qu'outil de démêlement du cours des choses; tandis qu'il conduira à faire apparaître la validité (régularité) ou la nonvalidité (irrégularité) de la réalité elle-même, de la réalisation ou conduite humaine encadrée par la règle éthique, et indirectement à donner la mesure de l'effectivité de celle-ci, c'est-à-dire la mesure dans laquelle cette règle est effectivement suivie et remplit sa fonction directive.

5) Ce sont aussi des vues négationnistes à l'égard des catégories modales dans le domaine du droit et de l'éthique qui caractérisent le réalisme scandinave. Ce courant de pensée juridique est très proche, sur ce point, du positivisme logique ${ }^{47}$. Il a pris, en effet, ses racines dans un mouvement philosophique plus général connu sous l'étiquette "École d'Uppsala » créé au début du $\mathrm{XX}^{\mathrm{e}}$ siècle par le philosophe suédois Axel Hägerström, et qui professait précisément des idées tout à fait similaires à celles soutenues à la même époque par le Cercle de Vienne et devenues ensuite les bases du positivisme logique : seul ce qui est réel et vérifiable expérimentalement peut faire l'objet d'une connaissance et donner lieu à des énoncés théoriques sensés ; les énoncés autres que descriptifs de réalités empiriques sont des énoncés métaphysiques dépourvus de sens et qu'une analyse logique vigilante doit débusquer et démonétiser ; il peut s'agir aussi de simples expressions de sentiments, — c'est le cas pour les énoncés de valeur. L'École d'Uppsala n'a guère prospéré, dans la philosophie générale scandinave, au-delà des années 1940 ; elle a eu, en revanche, des prolongements durables au niveau de la philosophie du droit scandinave à travers le mouvement réaliste qu'elle a inspiré et qu'on présente d'ailleurs comme "sa branche juridique " ${ }^{48}$. Hägerström lui-même avait déjà appliqué, en effet, dans ses ouvrages, ses idées et ses mots d'ordre au domaine du droit; des juristes scandinaves se sont inscrits dans son sillage, en particulier les suédois Vilhelm Lundstedt et Karl Olivecrona, et le danois Alf Ross.

Partant du même principe selon lequel, en tant que « science » produisant des énoncés sensés, la théorie du droit ne doit étudier que des réalités empiriques ${ }^{49}$, ces réalistes ont tous été amenés à donner des règles juridiques une image déformée, occultant complètement la signification propre et le rôle

47. Encore qu'il présente aussi, on le verra, des ressemblances avec le réalisme américain.

48. S. Strömholm et H.H. Vogel, op. cit., p. 5.

49. Ce qu'exprime le titre-phare de l'ouvrage célèbre d'Olivecrona: Law as Fact, Stevens and Sons, Londres, 1939. Sur ce point, le réalisme scandinave se démarque des conceptions éthiques du positivisme logique et rejoint plutôt le réalisme américain : le droit n'est pas réduit à de simples expressions de sentiments, mais à des faits. Ross est particulièrement proche des réalistes américains puisque pour lui le droit en vigueur, c'est ce que les tribunaux font (c'est-à-dire décident) et que la science du droit a pour objet de prédire. 
spécifique des modalités déontiques. Pour eux, une norme de conduite, c'est simplement un énoncé linguistique par lequel le locuteur cherche à obtenir un comportement d'autrui en exerçant une pression de fait sur lui, et notamment en le menaçant de sanction s'il n'a pas ce comportement : c'est à cela que se réduirait la fonction de direction des conduites des règles juridiques et éthiques. Aucune importance, aucune épaisseur n'est reconnue aux catégories modales; il s'agirait de pseudo-concepts, de catégories fictives, vides de sens, puisque sans référents dans le monde réel ${ }^{50}$.

Tout essai de formuler, sur une base scientifique, l'hypothèse que le droit pourrait comporter des devoirs, autrement qu'en exerçant une pression de pur fait sur les hommes, est voué à l'échec. De tels essais ne sauraient aboutir qu'à des contradictions et à d'autres erreurs. Nous voici donc à la frontière entre réalisme et métaphysique, entre la science et le mysticisme dans l'explication de ce qu'est le droit ${ }^{51}$.

La notion de «droit» serait pareillement, en tant que telle, une idée creuse, sauf à la ramener à un simple fait, celui d'occuper une "position favorable ", spécialement de bénéficier de la protection des tribunaux dans l'accomplissement d'une action ou la disposition d'une chose:

Le droit subjectif se dérobe à toute tentative pour en fixer le contenu. On ne réussit jamais à le saisir. Pour cette raison, on incline facilement à se replier sur quelque chose de compréhensible et de manifeste. La position favorable en tant que fait se présente alors naturellement à l'esprit ${ }^{52}$.

La présentation la plus caricaturale du prétendu caractère superfétatoire et archaïque des modalités déontiques, on la trouve dans une curieuse étude d'Alf Ross intitulée "Tû-Tû ${ }^{53}$. Il y est question d'une tribu vivant dans les îles Noïsulli au milieu du Pacifique sud, la tribu des Noît-cif qui compte parmi les peuples humains les plus primitifs actuellement au monde. Selon le rapport d'un anthropologue, les membres de cette tribu emploient, dans les cas d'infractions à divers tabous, un mot bizarre, le mot th-tû. Par exemple, «si un homme a une relation avec sa belle-mère, ou si un animal totem est tué, ou si quelqu'un a mangé une nourriture préparée pour le chef [...] les membres de la tribu disent que celui qui a commis cette infraction est devenu $t \hat{u}$ $t \hat{u} »$; et dans chacun de ces cas, une même conséquence est tirée : "celui qui est devenu tû-tû doit être soumis à une cérémonie spéciale de purification. »

50. Plus généralement, « les notions de justice, de droits subjectifs, d'obligations, de responsabilité sont dépourvues de sens », soutenait Vilhelm Lundstedt (1882-1995), le plus extrêmiste des réalistes scandinaves dans Le défaut de caractère scientifique de la science juridique (19321936). Ces concepts ne peuvent pas être objet de science » (J.C. Billier et A. Maryioli, Histoire de la philosophie du droit, Armand Colin, 2001, p. 185).

51. Karl Olivecrona, cité par S. Strömholm et H.H. Vogel (op. cit., p. 54).

52. Karl Olivecrona, cité par S. Strömholm et H.H. Vogel (op. cit., p. 56).

53. Harvard Law Review, vol. 70, 1956-1957, p. 812 s. (cette étude se trouve partiellement reproduite en traduction française dans l'ouvrage de M. Troper, F. Michaut et C. Grzegorczyk, Le positivisme juridique, Story Scientia - L.G.D.J., 1992, p. 207 s. : les citations du texte en sont extraites). 
Il est évident, pour Ross, que "tĥ-tû n'est rien du tout; c'est un mot dépourvu de toute espèce de signification ». Il aurait seulement pour fonction de permettre une espèce de décomposition des énoncés normatifs. Ross cite ainsi ces deux phrases entendues et rapportées par l'anthropologue:

1. "Si quelqu'un a mangé la nourriture du chef, il est th-th.»

2. «Si quelqu'un est $t \hat{u}-t \hat{u}$, il sera soumis à une cérémonie de purification.»

On pourrait parfaitement, observe Ross, supprimer cet intermédiaire ou

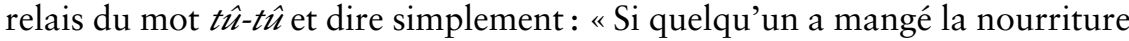
du chef, il sera soumis à une cérémonie de purification. "Selon le penseur danois, les «droits» et «obligations » dont on parle dans le champ juridique seraient d'exacts équivalents du mot $t \hat{u}$ - $t \hat{u}$, des termes vides de sens dont on pourrait en théorie pareillement se passer. Il prend l'exemple de ces deux propositions :

1. "Si un prêt a été accordé, alors naît une créance " (on pourrait dire aussi une dette, une obligation).

2. "Si une créance ou une dette existe, alors le paiement sera effectué le jour de l'échéance. »

On pourrait faire abstraction des mots "créance ", "dette ", "obligation ", et dire simplement : "Si un prêt a été accordé, alors le paiement (plus exactement le remboursement) sera effectué le jour de l'échéance» — cette proposition signifiant plus précisément, selon la conception « judiciaire » du droit de Ross, que « le tribunal condamnera l'emprunteur au remboursement à compter du jour de l'échéance ". Il serait ainsi possible, théoriquement, de se passer des termes modaux (dont on voit, à travers cet exemple, que Ross s'est de toute façon débarrassé de la signification logique pour leur faire signifier un pur fait). Théoriquement, car en pratique, prétend Ross, il faudrait alors alourdir considérablement les énoncés juridiques, ce qui serait très peu commode: il faudrait, pour chaque fait générateur de dette, détailler les conséquences juridiques qui se trouvent impliquées derrière ce terme ("si un dommage est causé, alors le tribunal condamnera son auteur à payer des dommages et intérêts à la victime à compter de la survenance de ce dommage ", «si la vente d'une chose a lieu, alors le tribunal condamnera l'acheteur à payer le prix à compter de la remise de la chose ", "si un appartement est loué, alors le tribunal condamnera le locataire à payer le loyer aux termes échus ", etc.). On obtient, au contraire une avantageuse simplification en disant que le dommage, la vente ou la location sont générateurs de « dettes » et en énonçant par ailleurs, de manière synthétique, que « si une dette existe, le tribunal condamnera le débiteur au paiement au jour de l'échéance ". Voilà à quoi se réduirait la fonction des droits et obligations: ce seraient bien des héritiers du $t \hat{u}$-tû des Noît-cif, qui remplissait déjà la même fonction - des espèces de placebos langagiers. 
On voit, au terme de ce panorama - et ce sera la conclusion de mon étude - , que la recherche ontologique dans le domaine du droit et plus généralement de l'éthique, mais aussi dans celui de la science, a encore bien du chemin à parcourir avant de parvenir à dégager et à enraciner fermement dans les esprits une vue claire des règles - pratiques et théoriques - et de leur mode de fonctionnement. Dans ces domaines, la fameuse maxime gravée sur le fronton du temple d'Apollon à Delphes continue de nous lancer son lancinant défi : «Connais-toi toi-même. » 